\title{
THE EQUIVARIANT BRAUER GROUPS OF COMMUTING FREE AND PROPER ACTIONS ARE ISOMORPHIC
}

\author{
ALEXANDER KUMJIAN, IAIN RAEBURN, AND DANA P. WILLIAMS
}

(Communicated by Palle E. T. Jorgensen)

Abstract. If $X$ is a locally compact space which admits commuting free and proper actions of locally compact groups $G$ and $H$, then the Brauer groups $\operatorname{Br}_{H}(G \backslash X)$ and $\operatorname{Br}_{G}(X / H)$ are naturally isomorphic.

Rieffel's formulation of Mackey's Imprimitivity Theorem asserts that if $H$ is a closed subgroup of a locally compact group $G$, then the group $C^{*}$-algebra $C^{*}(H)$ is Morita equivalent to the crossed product $C_{0}(G / H) \rtimes G$. Subsequently, Rieffel found a symmetric version, involving two subgroups of $G$, and Green proved the following Symmetric Imprimitivity Theorem: If two locally compact groups act freely and properly on a locally compact space $X, G$ on the left and $H$ on the right, then the crossed products $C_{0}(G \backslash X) \rtimes H$ and $C_{0}(X / H) \rtimes G$ are Morita equivalent. (For a discussion and proofs of these results, see [15].) Here we shall show that in this situation there is an isomorphism $\mathrm{Br}_{H}(G \backslash X) \cong \mathrm{Br}_{G}(X / H)$ of the equivariant Brauer groups introduced in [2].

Suppose $(G, X)$ is a second countable locally compact transformation group. The objects in the underlying set $\mathfrak{B r}_{G}(X)$ of the equivariant $\operatorname{Brauer}_{\text {group }} \operatorname{Br}_{G}(X)$ are dynamical systems $(A, G, \alpha)$, in which $A$ is a separable continuous-trace $C^{*}$-algebra with spectrum $X$, and $\alpha: G \rightarrow \operatorname{Aut}(A)$ is a strongly continuous action of $G$ on $A$ inducing the given action of $G$ on $X$. The equivalence relation on such systems is the equivariant Morita equivalence studied in [1], [3]. The group operation is given by $[A, \alpha] \cdot[B, \beta]=\left[A \otimes_{C(X)} B, \alpha \otimes \beta\right]$, the inverse of $[A, \alpha]$ is the conjugate system $[\bar{A}, \bar{\alpha}]$, and the identity is represented by $\left(C_{0}(X), \tau\right)$, where $\tau_{s}(f)(x)=f\left(s^{-1} \cdot x\right)$.

Notation. Suppose that $H$ is a locally compact group, that $X$ is a free and proper right $H$-space, and that $(B, H, \beta)$ is a dynamical system. Then $\operatorname{Ind}_{H}^{X}(B, \beta)$ will be the $C^{*}$-algebra (denoted by $G C(X, B)^{\alpha}$ in [13] and by $\operatorname{Ind}(B ; X, H, \beta)$ in [11]) of bounded continuous functions $f: X \rightarrow B$ such that $\beta_{h}(f(x \cdot h))=f(x)$, and $x \cdot H \mapsto\|f(x)\|$ belongs to $C_{0}(X / H)$.

We now state our main theorem.

Received by the editors August 30, 1994

1991 Mathematics Subject Classification. Primary 46L05, 46L35.

Key words and phrases. Crossed product, continuous-trace, $C^{*}$-algebra, Morita equivalence.

The third author was partially supported by the National Science Foundation.

This research was supported by the Australian Department of Industry, Science, and Technology. 
Theorem 1. Let $X$ be a second countable locally compact Hausdorff space, and let $G$ and $H$ be second countable locally compact groups. Suppose that $X$ admits a free and proper left $G$-action, and a free and proper right $H$-action such that $(g \cdot x) \cdot h=g \cdot(x \cdot h)$ for all $x \in X, g \in G$, and $h \in H$. Then there is an isomorphism $\Theta$ of $\mathrm{Br}_{H}(G \backslash X)$ onto $\mathrm{Br}_{G}(X / H)$ satisfying:

(1) if $(A, \alpha)$ represents $\Theta[B, \beta]$, then $A \rtimes_{\alpha} G$ is Morita equivalent to $B \rtimes_{\beta} H$;

(2) $\Theta[B, \beta]$ is realised by the pair $\left.\operatorname{Ind}_{H}^{X}(B, \beta) / J, \tau \otimes \mathrm{id}\right)$ in $\mathfrak{B r}_{G}(X / H)$, where $\tau \otimes \mathrm{id}$ denotes left translation and, if $\pi_{G \cdot x}$ is the element of $\widehat{B}=G \backslash X$ corresponding to $G \cdot x$,

$$
J=\left\{f \in \operatorname{Ind}_{H}^{X}(B, \beta): \pi_{G \cdot x}(f(x))=0 \text { for all } x \in X\right\} .
$$

Item (1) is itself a generalization of Green's symmetric imprimitivity theorem, and our proof of Theorem 1 follows the approach to Green's theorem taken in [3]: prove that both $C_{0}(G \backslash X) \rtimes H$ and $C_{0}(X / H) \rtimes G$ are Morita equivalent to $C_{0}(X) \rtimes_{\alpha}(G \times H)$, where $\alpha_{s, h}(f)(x)=f\left(s^{-1} \cdot x \cdot h\right)$, by noting that the Morita equivalences of $C_{0}(X) \rtimes G$ with $C_{0}(G \backslash X)$ and $C_{0}(X) \rtimes H$ with $C_{0}(X / H)$ ([7], [15, Situation 10]) are equivariant, and hence induce Morita equivalences

$$
\begin{aligned}
C_{0}(G \backslash X) \rtimes H & \sim\left(C_{0}(X) \rtimes G\right) \rtimes H \cong C_{0}(X) \rtimes(G \times H) \\
& \cong\left(C_{0}(X) \rtimes H\right) \rtimes G \sim C_{0}(X / H) \rtimes G .
\end{aligned}
$$

The same symmetry considerations show that it will be enough to prove that $\operatorname{Br}_{H}(G \backslash X) \cong \operatorname{Br}_{G \times H}(X)$. Since we already know that $\operatorname{Br}(G \backslash X) \cong \operatorname{Br}_{G}(X)[2$, $\S 6.2$, we just have to check that this isomorphism is compatible with the actions of $H$.

Suppose $G$ acts freely and properly on $X$, and $p: X \rightarrow G \backslash X$ is the orbit map. If $B$ is a $C^{*}$-algebra with a nondegenerate action of $C_{0}(G \backslash X)$, then the pull-back $p^{*} B$ is the quotient of $C_{0}(X) \otimes B$ by the balancing ideal

$$
I_{G \backslash X}=\overline{\operatorname{span}}\left\{f \cdot \phi \otimes b-\phi \otimes f \cdot b: \phi \in C_{0}(X), f \in C_{0}(G \backslash X), b \in B\right\} ;
$$

in other words, $p^{*} B=C_{0}(X) \otimes_{C(G \backslash X)} B$. The nondegenerate action of $C_{0}(G \backslash X)$ on $B$ induces a continuous map $q$ of $\widehat{B}$ onto $G \backslash X$, characterized by $\pi(f \cdot b)=$ $f(q(\pi)) \pi(b)$. Then under the natural identification of $C_{0}(X) \otimes B$ with $C_{0}(X, B)$,

$$
I_{G \backslash X} \cong\left\{f \in C_{0}(X, B): \pi(f(x))=0 \text { for all } x \in q(\pi)\right\},
$$

so that $p^{*} B$ has spectrum

$$
\widehat{p^{*} B}=\{(x, \pi) \in X \times \widehat{B}: G \cdot x=q(\pi)\} .
$$

If $B$ is a continuous-trace algebra with spectrum $G \backslash X$, then $p^{*} B$ is a continuoustrace algebra with spectrum $X$.

The isomorphism $\Theta: \operatorname{Br}(G \backslash X) \cong \operatorname{Br}_{G}(X)$ is given by $\Theta[A]=\left[p^{*} A, \tau \otimes \mathrm{id}\right]$. To prove $\Theta$ is surjective in [2], we used [12, Theorem 1.1], which implies that if $(B, \beta) \in \mathfrak{B r}_{G}(X)$, then $B \rtimes_{\beta} G$ is a continuous-trace algebra with spectrum $G \backslash X$ such that $(B, \beta)$ is Morita equivalent to $\left(p^{*}\left(B \rtimes_{\beta} G\right), \tau \otimes \mathrm{id}\right)$, and hence that $[B, \beta]=\Theta\left[B \rtimes_{\beta} G\right.$, id]. In obtaining the required equivariant version of $[12$, Theorem 1.1], we have both simplified the proof and mildly strengthened the conclusion (see Corollary 4 below). However, with all these different group actions around, the notation could get messy, and we pause to establish some conventions. 
Notation. We shall be dealing with several spaces carrying a left action of $G$ and/or a right action of $H$. We denote by $\tau$ the action of $G$ by left translation on $C_{0}(G)$, $C_{0}(X)$ or $C_{0}(G \backslash X)$, and by $\sigma$ any action of $H$ by right translation; we shall also use $\sigma^{G}$ to denote the action of $G$ by right translation on $C_{0}(G)$. Restricting an action $\beta$ of $G \times H$ on an algebra $A$ gives actions $\alpha: G \rightarrow \operatorname{Aut}(A), \gamma: H \rightarrow \operatorname{Aut}(A)$ such that

$$
\alpha_{s}\left(\gamma_{h}(a)\right)=\gamma_{h}\left(\alpha_{s}(a)\right) \quad \text { for all } h \in H, s \in G, a \in A .
$$

Conversely, two actions $\alpha, \gamma$ satisfying (1) define an action of $G \times H$ on $A$, which we denote by $\alpha \gamma$; we write $\gamma$ for id $\gamma$ since it will be clear from context whether an action of $H$ or $G \times H$ is called for. If $\Phi:(A, G, \alpha) \rightarrow(B, G, \beta)$ is an equivariant isomorphism (i.e. $\Phi\left(\alpha_{s}(a)\right)=\beta_{s}(\Phi(a))$ ), then we denote by $\Phi \rtimes$ id the induced isomorphism of $A \rtimes_{\alpha} G$ onto $B \rtimes_{\beta} G$. Similarly, if $\alpha$ and $\gamma$ satisfy (1), we write $\alpha \rtimes$ id for the induced action of $G$ on $A \rtimes_{\gamma} H$.

Lemma 2. Suppose a locally compact group $G$ acts freely and properly on a locally compact space $X$, and that $A$ is a $C^{*}$-algebra carrying a non-degenerate action of $C_{0}(X)$. If $\alpha: G \rightarrow \operatorname{Aut}(A)$ is an action of $G$ on $A$ satisfying $\alpha_{s}(\phi \cdot a)=\tau_{s}(\phi) \cdot \alpha_{s}(a)$, then the map sending $f \otimes a$ in $C_{0}(X) \otimes A$ to the function $s \mapsto f \cdot \alpha_{s}^{-1}(a)$ induces an equivariant isomorphism $\Phi$ of $\left(C_{0}(X) \otimes_{C(G \backslash X)} A, G\right.$, id $\left.\otimes \alpha\right)$ onto $\left(C_{0}(G, A), G, \tau \otimes\right.$ id).

Remark 3. For motivation, consider the case where $A=C_{0}(X)$. Then the map $\Psi: C_{b}(X \times X) \rightarrow C_{b}(G \times X)$ defined by $\Psi(f)(s, x)=f(x, s \cdot x)$ maps $C_{0}$ to $C_{0}$ precisely when the action is proper, has range which separates the points of $G \times Y$ precisely when the action is free, and has kernel consisting of the functions which vanish on the closed subset $\Delta=\{(x, y): G \cdot x=G \cdot y\}$. Thus the free and proper actions are precisely those for which $\Psi$ induces an isomorphism of $C_{0}(X) \otimes_{C(G \backslash X)} C_{0}(X)$ onto $C_{0}(G) \otimes C_{0}(X)$.

Proof of Lemma 2. If $\phi \in C_{0}(G \backslash X)$, then $f \cdot \phi \otimes a$ and $f \otimes \phi \cdot a$ have the same image in $C_{0}(G, A)$, and the map factors through the balanced tensor product as claimed. Further, $\Phi$ is related to the map $\Psi$ in Remark 3 by

$$
\Phi(f \otimes g \cdot a)=(\Psi(f \otimes g)(s, \cdot)) \cdot \alpha_{s}^{-1}(a) .
$$

Thus it follows from the remark that (2) defines an element of $C_{0}(G, A)$ and that the closure of the range of $\Phi$ contains all functions of the form $s \mapsto \xi(s) f \cdot \alpha_{s}^{-1}(a)$ for $\xi \in C_{c}(G), f \in C_{c}(X)$, and $a \in A$. These elements span a dense subset of $C_{0}(G, A)$, and hence $\Phi$ is surjective. The nondegenerate action of $C_{0}(X)$ on $A$ induces a continuous equivariant map $q$ of $\widehat{A}$ onto $X$ such that $\pi(f \cdot a)=f(q(\pi)) \pi(a)$, and the balanced tensor product $C_{0}(X) \otimes_{C(G \backslash X)} A$ has spectrum $\Delta=\{(x, \pi): G \cdot x=$ $G \cdot q(\pi)\}$. Since each representation $(q(\pi), s \cdot \pi)=\left(q(\pi), \pi \circ \alpha_{s}^{-1}\right)$ in $\Delta$ factors through $\Phi$ and the representation $b \mapsto \pi(b(s))$ of $C_{0}(G, A)$, the homomorphism $\Phi$ is also injective. Finally, to see the equivariance, we compute:

$$
\begin{aligned}
\Phi\left(\mathrm{id} \otimes \alpha_{s}(h \otimes a)\right)(t) & =h \cdot \alpha_{t}^{-1}\left(\alpha_{s}(a)\right)=\Phi(h \otimes a)\left(s^{-1} t\right) \\
& =\tau_{s} \otimes \operatorname{id}(\Phi(h \otimes a))(t) . \quad \square
\end{aligned}
$$

Corollary 4 (cf. [12, Theorem 1.1]). Let $(G, X)$ and $\alpha: G \rightarrow \operatorname{Aut}(A)$ be as in Lemma 2. Then there is an equivariant isomorphism of $\left(p^{*}\left(A \rtimes_{\alpha} G\right), G, p^{*}\right.$ id $)$ onto $\left(A \otimes \mathcal{K}\left(L^{2}(G)\right), G, \alpha \otimes \operatorname{Ad} \rho\right)$. 
Proof. A routine calculation shows that the equivariant isomorphism $\Phi$ of Lemma 2 gives an equivariant isomorphism

$$
\begin{aligned}
\Phi \rtimes \mathrm{id}:\left(\left(C_{0}(X) \otimes_{C(G \backslash X)} A\right)\right. & \left.\rtimes_{\mathrm{id} \otimes \alpha} G,(\tau \otimes \mathrm{id}) \rtimes \mathrm{id}\right) \\
& \rightarrow\left(C_{0}(G, A) \rtimes_{\tau \otimes \mathrm{id}} G,\left(\sigma^{G} \otimes \alpha\right) \rtimes \mathrm{id}\right) .
\end{aligned}
$$

We also have equivariant isomorphisms

$$
\begin{aligned}
\left(C_{0}(G, A) \rtimes_{\tau \otimes \mathrm{id}} G,\left(\sigma^{G} \otimes \alpha\right) \rtimes \mathrm{id}\right) & \cong\left(A \otimes\left(C_{0}(G) \rtimes_{\tau} G\right), \alpha \otimes\left(\sigma^{G} \rtimes \mathrm{id}\right)\right), \\
& \cong\left(A \otimes \mathcal{K}\left(L^{2}(G)\right), \alpha \otimes \operatorname{Ad} \rho\right)
\end{aligned}
$$

and

$(5)$

$\left(C_{0}(X) \otimes_{C(G \backslash X)}\left(A \rtimes_{\alpha} G\right), \tau \otimes \mathrm{id}\right) \cong\left(\left(C_{0}(X) \otimes_{C(G \backslash X)} A\right) \rtimes_{\mathrm{id} \otimes \alpha} G,(\tau \otimes \mathrm{id}) \rtimes \mathrm{id}\right) ;$ combining (3), (4), and (5) gives the result.

Lemma 5. In addition to the hypotheses of Lemma 2, suppose that $H$ is a locally compact group acting on the right of $X$, and that $(A, H, \gamma)$ is a dynamical system such that $\alpha$ and $\gamma$ commute and $\gamma_{h}(f \cdot a)=\sigma_{h}(f) \cdot \gamma_{h}(a)$ for $h \in H, f \in C_{0}(X)$, $a \in A$. Then the action $\tau \sigma \otimes \gamma$ of $G \times H$ on $C_{0}(X) \otimes A$ preserves the balancing ideal $I_{G \backslash X}$, and hence induces an action of $G \times H$ on $C_{0}(X) \otimes_{C(G \backslash X)} A$, also denoted $\tau \sigma \otimes \gamma$. The equivariant isomorphism of Lemma 2 induces an equivariant isomorphism

$$
\begin{gathered}
\left(\left(C_{0}(X) \otimes_{C(G \backslash X)} A\right) \rtimes_{\mathrm{id} \otimes \alpha} G,(\tau \sigma \otimes \gamma) \rtimes \mathrm{id}\right) \\
\cong\left(C_{0}(G, A) \rtimes_{\tau \otimes \mathrm{id}} G,\left(\sigma^{G} \otimes \alpha \gamma\right) \rtimes \mathrm{id}\right) .
\end{gathered}
$$

Proof. The first assertion is straightforward. For the second, we can consider the actions of $H$ and $G$ separately. We have already observed in (3) that $\Phi \rtimes$ id intertwines the $G$-actions. On the other hand, if $h \in H$ and $t \in G$, then

$$
\begin{aligned}
\Phi\left(\sigma_{h} \otimes \gamma_{h}(f \otimes a)\right)(t) & =\sigma_{h}(f) \cdot \alpha_{t}^{-1}\left(\gamma_{h}(a)\right)=\sigma_{h}(f) \cdot \gamma_{h}\left(\alpha_{t}^{-1}(a)\right) \\
& =\gamma_{h}(\Phi(f \otimes a)(t)) . \quad \square
\end{aligned}
$$

Corollary 6. Let ${ }_{G} X_{H}$ and $\alpha: G \rightarrow \operatorname{Aut}(A), \gamma: H \rightarrow \operatorname{Aut}(A)$ be as in the lemma. Denote by $p$ the orbit map of $X$ onto $G \backslash X$. Then there is an equivariant isomorphism

$$
\left(p^{*}\left(A \rtimes_{\alpha} G\right), G \times H, \tau \sigma \otimes(\gamma \rtimes \mathrm{id})\right) \cong\left(A \otimes \mathcal{K}\left(L^{2}(G)\right), G \times H, \alpha \gamma \otimes \operatorname{Ad} \rho\right) .
$$

Proof. Compose the isomorphism of Lemma 5 with (4) and (5).

We are now ready to define our map of $\operatorname{Br}_{H}(G \backslash X)$ into $\operatorname{Br}_{G \times H}(X)$. Suppose $(B, \beta) \in \mathfrak{B r}_{H}(X)$. Then the action $\tau \sigma \otimes \beta$ of $G \times H$ preserves the balancing ideal $I_{G \backslash X}$ : if $\phi \in C_{0}(G \backslash X)$, then

$$
\begin{gathered}
(\tau \sigma \otimes \beta)_{s, h}(f \cdot \phi \otimes b-f \otimes \phi \cdot b)=\sigma_{h}\left(\tau_{s}(f \cdot \phi)\right) \otimes \beta_{h}(b)-\sigma_{h}\left(\tau_{s}(f)\right) \otimes \beta_{h}(\phi \cdot b) \\
=\sigma_{h}\left(\tau_{s}(f)\right) \cdot \sigma_{h}(\phi) \otimes \beta_{h}(b)-\sigma_{h}\left(\tau_{s}(f)\right) \otimes \sigma_{h}(\phi) \cdot \beta_{h}(b) .
\end{gathered}
$$

Since $p^{*}(B)$ is a continuous-trace $C^{*}$-algebra with spectrum $X$ [12, Lemma 1.2], and $\tau \sigma \otimes \beta$ covers the canonical $G \times H$-action on $X$, we can define $\theta: \mathfrak{B r}_{H}(G \backslash X) \rightarrow$ $\mathfrak{B r}_{G \times H}(X)$ by $\theta(B, \beta)=\left(p^{*}(B), \tau \sigma \otimes \beta\right)$.

Similarly if $(A, \alpha \gamma) \in \mathfrak{B r}_{G \times H}(X)$, then $A \rtimes_{\alpha} G$ is a continuous-trace $C^{*}$-algebra with spectrum $G \backslash X$ by [12, Theorem 1.1]. Since $\gamma$ is compatible with $\sigma$, we have $\gamma_{h}(\phi \cdot z(s))=\sigma_{h}(\phi) \cdot \gamma_{h}(z(s))$ for $z \in C_{c}(G, A)$, and hence $\gamma \rtimes$ id covers the 
given action of $H$ on $X$. Thus we can define $\lambda: \mathfrak{B r}_{G \times H}(X) \rightarrow \mathfrak{B r}_{H}(G \backslash X)$ by $\lambda(A, \alpha \gamma)=\left(A \rtimes_{\alpha} G, \gamma \rtimes \mathrm{id}\right)$.

Proposition 7. Let $X$ be a second countable locally compact Hausdorff space, and let $G$ and $H$ be second countable locally compact groups. Suppose that $X$ admits a free and proper left $G$-action, and an $H$-action such that $(g \cdot x) \cdot h=g \cdot(x \cdot h)$ for all $x \in X, g \in G$, and $h \in H$. Then $\theta$ and $\lambda$ above preserve Morita equivalence classes, and define homomorphisms $\Theta: \operatorname{Br}_{H}(G \backslash X) \rightarrow \operatorname{Br}_{G \times H}(X)$ and $\Lambda: \operatorname{Br}_{G \times H}(X) \rightarrow$ $\operatorname{Br}_{H}(G \backslash X)$. In fact, $\Theta$ is an isomorphism with inverse $\Lambda$, and if $\Theta[B, \beta]=[A, \alpha]$, then $B \rtimes_{\beta} H$ is Morita equivalent to $A \rtimes_{\alpha}(G \times H)$.

Proof. If $(\mathcal{Y}, v)$ implements an equivalence between $(B, \beta)$ and $\left(B^{\prime}, \beta^{\prime}\right)$ in $\mathfrak{B r}_{H}(G \backslash X)$, then the external tensor product $\mathcal{Z}=C_{0}(X) \widehat{\otimes} \mathcal{Y}$, as defined in $[9$, $\S 1.2]$ or $[2, \S 2]$, is a $C_{0}(X) \otimes B-C_{0}(X) \otimes B^{\prime}$-imprimitivity bimodule. A routine argument, similar to that in [2, Lemma 2.1], shows that the Rieffel correspondence [14, Theorem 3.1] between the lattices of ideals in $C_{0}(X) \otimes B$ and in $C_{0}(X) \otimes B^{\prime}$ maps the balancing ideal $I=I_{C(G \backslash X)}$ in $C_{0}(X) \otimes B$ to the balancing ideal $J=J_{C(G \backslash X)}$ in $C_{0}(X) \otimes B^{\prime}$. Thus $\left[14\right.$, Corollary 3.2] implies that $\mathcal{X}=\mathcal{Z} / \mathcal{Z} \cdot J$ is a $p^{*}(B)-p^{*}\left(B^{\prime}\right)$ imprimitivity bimodule. Since $f \cdot x=x \cdot f$ for all $x \in \mathcal{X}$ and $f \in C_{0}(X)$, it follows from [10, Proposition 1.11] that $\mathcal{X}$ implements a Morita equivalence over $X$. More tedious but routine calculations show that the map defined on elementary tensors in $\mathcal{Z}_{0}=C_{0}(X) \odot \mathcal{Y}$ by $u_{(s, h)}^{0}(f \otimes y)=\sigma_{h}\left(\tau_{s}(f)\right) \otimes v_{h}(y)$ extends to the completion $\mathcal{Z}$, and defines a strongly continuous map $u: G \times H \rightarrow \operatorname{Iso}(\mathcal{X})$ such that $(\mathcal{X}, u)$ implements an equivalence between $\left(p^{*}(B), \tau \sigma \otimes \beta\right)$ and $\left(p^{*}\left(B^{\prime}\right), \tau \sigma \otimes \beta^{\prime}\right)$. Thus $\Theta$ is well defined.

Observe that

$$
\begin{aligned}
\Theta\left([B, \beta]\left[B^{\prime}, \beta^{\prime}\right]\right) & =\Theta\left(\left[B \otimes_{C(G \backslash X)} B^{\prime}, \beta \otimes \beta^{\prime}\right]\right) \\
& =\left[p^{*}\left(B \otimes_{C(G \backslash X)} B^{\prime}\right), \tau \sigma \otimes\left(\beta \otimes \beta^{\prime}\right)\right] .
\end{aligned}
$$

But (6) is the class of

$$
\begin{aligned}
\left(C_{0}(X)\right. & \left.\otimes_{C(G \backslash X)} B \otimes_{C(G \backslash X)} B^{\prime}, \tau \sigma \otimes \beta \otimes \beta^{\prime}\right) \\
& \sim\left(C_{0}(X) \otimes_{C(X)} C_{0}(X) \otimes_{C(G \backslash X)} B \otimes_{C(G \backslash X)} B^{\prime}, \tau \sigma \otimes \tau \sigma \otimes \beta \otimes \beta^{\prime}\right) \\
& \sim\left(C_{0}(X) \otimes_{C(G \backslash X)} B \otimes_{C(X)} C_{0}(X) \otimes_{C(G \backslash X)} B^{\prime}, \tau \sigma \otimes \beta \otimes \tau \sigma \otimes \beta^{\prime}\right),
\end{aligned}
$$

which represents the product of $\Theta[B, \beta]$ and $\Theta\left[B^{\prime}, \beta^{\prime}\right]$. Thus $\Theta$ is a homomorphism.

Now suppose that $(A, \alpha \gamma) \sim\left(A^{\prime}, \alpha^{\prime} \gamma^{\prime}\right)$ in $\mathfrak{B r}_{G \times H}(X)$ via $(\mathcal{Z}, w)$. Then $u_{s}=$ $w_{(s, e)}$ and $v_{h}=w_{(e, h)}$ define actions of $G$ and $H$, respectively, on $\mathcal{Z}$. In particular, $(\mathcal{Z}, u)$ implements an equivalence between $(A, \alpha)$ and $\left(A^{\prime}, \alpha^{\prime}\right)$ in $\mathfrak{B r}_{G}(X)$. It follows from $[1, \S 6]$ that $\mathcal{Y}_{0}=C_{c}(G, \mathcal{Z})$ can be completed to a $A \rtimes_{\alpha} G-A^{\prime} \rtimes_{\alpha^{\prime}} G$ imprimitivity bimodule $\mathcal{Y}$. One can verify that the induced $C_{0}(G \backslash X)$-actions on $\mathcal{Y}_{0}$ are given by $(\phi \cdot x)(t)=\phi \cdot(x(t))$ and $(x \cdot \phi)(t)=(x(t)) \cdot \phi$, and [10, Proposition 1.11] implies that $\mathcal{Y}$ is an imprimitivity bimodule over $G \backslash X$. Now define $\tilde{v}_{h}^{0}$ on $\mathcal{Y}_{0}$ by $\tilde{v}_{h}^{0}(x)(t)=v_{h}(x(t))$. Using the inner products defined in $[1, \S 6]$,

$$
\begin{aligned}
A \rtimes_{\alpha} G\left\langle\tilde{v}_{h}^{0}(x), \tilde{v}_{h}^{0}(y)\right\rangle(t) & =\int_{G^{A}}\left\langle\tilde{v}_{h}^{0}(x)(s), \Delta\left(t^{-1} s\right) u_{t}\left(\tilde{v}_{h}^{0}(y)\left(t^{-1} s\right)\right)\right\rangle d s \\
& =\int_{G^{A}}\left\langle v_{h}(x(s)), \Delta\left(t^{-1} s\right) u_{t}\left(v_{h}\left(y\left(t^{-1} s\right)\right)\right)\right\rangle d s \\
& =\gamma_{h}\left(A \rtimes_{\alpha} G\langle x, y\rangle(t)\right),
\end{aligned}
$$


where, in the last equality, we use $u_{s} \circ v_{h}=v_{h} \circ u_{s}$. A similar computation shows that $\left\langle\tilde{v}_{h}^{0}(x), \tilde{v}_{h}^{0}(y)\right\rangle_{A^{\prime} \rtimes_{\alpha^{\prime}} G}(t)=\gamma_{h}^{\prime}\left(\langle x, y\rangle_{A^{\prime} \rtimes_{\alpha^{\prime}} G}(t)\right)$. Thus $\tilde{v}_{h}^{0}$ extends to all of $\mathcal{Y}$ and defines a map $\tilde{v}: H \rightarrow \operatorname{Iso}(\mathcal{Y})$, and it is not hard to verify that $\tilde{v}$ is strongly continuous. Therefore $\left(A \rtimes_{\alpha} G, \gamma \rtimes\right.$ id $) \sim\left(A^{\prime} \rtimes_{\alpha^{\prime}} G, \gamma^{\prime} \rtimes\right.$ id $)$ in $\mathfrak{B r}_{G \times H}(X)$, and $\Lambda$ is well defined.

Now it will suffice to show that, for $\mathfrak{a} \in \mathfrak{B r}_{H}(G \backslash X)$ and $\mathfrak{b} \in \mathfrak{B r}_{G \backslash H}(X)$, $\theta(\lambda(\mathfrak{b})) \sim \mathfrak{b}$ and $\lambda(\theta(\mathfrak{a})) \sim \mathfrak{a}$. For the first of these, suppose that $(A, \alpha \gamma) \in$ $\mathfrak{B r}_{G \times H}(X)$. Then $\theta(\lambda(A, \alpha \gamma))=\left(p^{*}\left(A \rtimes_{\alpha} G\right),(\tau \sigma \otimes \gamma) \rtimes \mathrm{id}\right)$, which by Corollary 6 is equivalent to $\left(A \otimes \mathcal{K}\left(L^{2}(G)\right), \alpha \gamma \otimes \operatorname{Ad} \rho\right)$, and hence to $(A, \alpha \gamma)$. For the other direction, suppose that $(B, \beta) \in \mathfrak{B r}_{H}(G \backslash X)$. Then $\lambda(\theta(B, \beta))=\left(p^{*} B \rtimes_{\tau \otimes \text { id }} G\right.$, $(\sigma \otimes \beta) \rtimes \mathrm{id})$. Now

$$
p^{*} B \rtimes_{\tau \otimes \mathrm{id}} G \cong\left(C_{0}(X) \otimes_{C(G \backslash X)} B\right) \rtimes_{\tau \otimes \mathrm{id}} G \cong\left(C_{0}(X) \rtimes_{\tau} G\right) \otimes_{C(G \backslash X)} B,
$$

which is Morita equivalent to $C_{0}(G \backslash X) \otimes_{C(G \backslash X)} B \cong B$. Because the Morita equivalence of $C_{0}(X) \rtimes G$ with $C_{0}(G \backslash X)$ is $H$-equivariant [3], it follows that

$\lambda(\theta(B, \beta))=\left(p^{*} B \rtimes_{\tau \otimes \text { id }} G,(\sigma \otimes \beta) \rtimes\right.$ id $) \sim\left(C_{0}(G \backslash X) \otimes_{C(G \backslash X)} B, \sigma \otimes \beta\right) \cong(B, \beta)$.

This shows that $\Lambda \circ \Theta$ is the identity, and also implies that

$$
p^{*} B \rtimes_{\tau \sigma \otimes \beta}(G \times H) \cong\left(p^{*} B \rtimes_{\tau \otimes \text { id }} G\right) \rtimes_{\sigma \otimes \beta} H \sim B \rtimes_{\beta} H,
$$

which proves the last assertion.

Remark 8. We showed that $\Lambda$ is a well-defined map of $\operatorname{Br}_{G \times H}(X)$ into $\operatorname{Br}_{H}(G \backslash X)$, and that it is a set-theoretic inverse for $\Theta$; since $\Theta$ is a group homomorphism, it follows that $\Lambda$ is also a homomorphism. This seems to be non-trivial: it implies that if $(A, \alpha),(B, \beta)$ are in $\mathfrak{B r}_{G}(X)$, then $\left(A \otimes_{C(X)} B\right) \rtimes_{\alpha \otimes \beta} G$ is Morita equivalent to $\left(A \rtimes_{\alpha} G\right) \otimes_{C(G \backslash X)}\left(B \rtimes_{\beta} G\right)$. We do not know what general mechanism is at work here. Certainly, it is a Morita equivalence rather than an isomorphism: if $G$ is finite and the algebra commutative, one algebra is $|G|$-homogeneous and the other $|G|^{2}$-homogeneous. The only direct way we have found uses [8, Theorem 17], which seems an excessively heavy sledgehammer.

Proof of Theorem 1. It follows from Proposition 7 that there are isomorphisms $\Theta_{H}: \operatorname{Br}_{H}(G \backslash X) \rightarrow \operatorname{Br}_{G \times H}(X)$ and $\Lambda_{G}: \operatorname{Br}_{G \times H}(X) \rightarrow \operatorname{Br}_{G}(X / H)$. Therefore $\Lambda_{G} \circ \Theta_{H}$ is an isomorphism of $\operatorname{Br}_{H}(G \backslash X)$ onto $\operatorname{Br}_{G}(X / H)$. Assertion (1) also follows from Proposition 7. The isomorphism $\Lambda_{G} \circ \Theta_{H}$ maps the class of $(B, \beta)$ in $\mathfrak{B r}_{H}(G \backslash X)$ to the class of $\left(p^{*}(B) \rtimes_{\sigma \otimes \beta} H,(\tau \otimes \mathrm{id}) \rtimes \mathrm{id}\right)$, so it remains to show that the latter is equivalent to $(A / J, \tau)$.

For convenience, write $I$ for the balancing ideal $I_{C(G \backslash X)}$ in $C_{0}(X) \otimes B$. Then

$$
p^{*}(B) \rtimes_{\sigma \otimes \beta} H=\left(\left(C_{0}(X) \otimes B\right) / I\right) \rtimes_{\sigma \otimes \beta} H=\left(C_{0}(X, B) \rtimes_{\sigma \otimes \beta} H\right) /\left(I \rtimes_{\sigma \otimes \beta} H\right)
$$

by, for example, [8, Proposition 12]. By [13, Theorem 2.2], $\mathcal{X}_{0}=C_{c}(X, B)$ can be completed to a $C_{0}(X, B) \rtimes_{\sigma \otimes \beta} H-A$-imprimitivity bimodule $\mathcal{X}$. The irreducible representations of $A$ are given by $M_{\left(x, \pi_{G} \cdot y\right)}(f)(x)=\pi_{G \cdot y}(f(x))[13$, Lemma 2.6]. In the proof of [13, Theorem 2.5], it was shown that the representation $\mathcal{X}^{M_{\left(x, \pi_{G} \cdot y\right)}}$ of $C_{0}(X, B) \rtimes_{\sigma \otimes \beta} H$ induced from $M_{\left(x, \pi_{G} \cdot y\right)}$ via $\mathcal{X}$ is equivalent to $\operatorname{Ind}_{\{e\}}^{G} N_{(x, G \cdot y)}$, where $N_{(x, G \cdot y)}$ is the analogous irreducible representation of $C_{0}(X, B)$. Since the orbit space for a proper action is Hausdorff, [5] implies that 
$\left(C_{0}(X, B), H, \sigma \otimes \beta\right)$ is regular. Since $R=\bigoplus_{x \in X} N_{(x, G \cdot x)}$ is a faithful representation of $p^{*}(B)$, it follows from [8, Theorem 24] that $\operatorname{Ind}_{\{e\}}^{G}(R)$ is a faithful representation of $p^{*}(B) \rtimes_{\sigma \otimes \beta} H$, and so has kernel $I \rtimes_{\sigma \otimes \beta} H$. On the other hand, $\operatorname{Ind}_{\{e\}}^{G}(R)$ is equivalent to $\bigoplus_{x \in X} \mathcal{X}^{M_{(x, G \cdot x)}}$. It follows from $[14, \S 3]$ that ${ }^{I} \mathcal{X}=\mathcal{X} / I \cdot \mathcal{X}$ is an $p^{*}(B) \rtimes_{\sigma \otimes \beta} H_{-X / H} A / J$-imprimitivity bimodule. Then the map $u_{s}^{0}: \mathcal{X}_{0} \rightarrow \mathcal{X}_{0}$ defined by $u_{s}^{0}(\xi)(x)=\xi\left(s^{-1} \cdot x\right)$ induces a map $u: G \rightarrow \operatorname{Iso}\left({ }^{I} \mathcal{X}\right)$ such that $\left({ }^{I} \mathcal{X}, u\right)$ implements the desired equivalence.

We close with two interesting special cases where the isomorphism takes a particularly elegant form. Recall that if $B$ is a continuous-trace $C^{*}$-algebra with spectrum $X$, then we may view $B$ as the sections $\Gamma_{0}(\xi)$ of a $C^{*}$-bundle $\xi$ vanishing at infinity.

Corollary 9. Suppose that $H$ is a closed subgroup of a second countable locally compact group $G$, and that $X$ is a second countable locally compact right $H$-space. Then $G \times X$ is a free and proper $H$-space via the diagonal action $(s, x) \cdot h=$ $(s h, x \cdot h)$. Thus $(G \times X) / H$ is a locally compact $G$-space via $s \cdot[r, x]=[s r, x]$, and the map $(B, \beta) \mapsto\left(\operatorname{Ind}_{H}^{G}(B, \beta), \tau\right)$ induces an isomorphism of $\operatorname{Br}_{H}(X)$ onto $\operatorname{Br}_{G}((X \times G) / H)$.

Proof. We apply Theorem 1 to ${ }_{G}(G \times X)_{H}$, where $G$ acts on the left of the first factor, obtaining an isomorphism of $\operatorname{Br}_{H}(X) \cong \operatorname{Br}_{H}(G \backslash(G \times X))$ onto $\operatorname{Br}_{G}((G \times X) / H)$ sending the class of $(B, \beta)$ to the class of $\operatorname{Ind}_{H}^{G \times X}(B, \beta) / J$ where $J=\{f: f(s, x)(x)=0\}$.

Given $f \in \operatorname{Ind}_{H}^{G \times X}(B, \beta)$ and $s \in G$, let $\Phi(f)(s)$ be the function from $X$ to $\xi$ defined by $\Phi(f)(s)(x)=f(s, x)(x)$. We claim $\Phi(f)(s) \in \Gamma_{0}(\xi)$. If $x_{0} \in X$, then $x \mapsto f\left(s, x_{0}\right)(x)$ is in $\Gamma_{0}(\xi)$, and $\left\|\Phi(s)(x)-f\left(s, x_{0}\right)(x)\right\|$ tends to zero as $x \rightarrow x_{0}$. It follows from [6, Proposition 1.6 (Corollary 1)] that $\Phi(f)(s)$ is continuous. To see that $\Phi(f)(s)$ vanishes at infinity, suppose that $\left\{x_{n}\right\} \subset X$ satisfies

$$
\left\|\Phi(f)(s)\left(x_{n}\right)\right\| \geq \varepsilon>0
$$

for all $n$. Then $\left\|f\left(s, x_{n}\right)\right\| \geq \varepsilon$ for all $n$, and passing to a subsequence and relabeling if necessary, there must be $h_{n} \in H$ such that $\left(s \cdot h_{n}, x_{n} \cdot h_{n}\right) \rightarrow(r, x)$. Then $h_{n} \rightarrow s^{-1} r \in H$, and $x_{n} \rightarrow x \cdot\left(r^{-1} s\right)$. In sum, $\Phi(f)(s) \in \Gamma_{0}(\xi)=B$. Now the continuity of $f$ easily implies that $s \mapsto \Phi(f)(s)$ is continuous from $G$ to $B$. Furthermore, since $\beta$ covers $\sigma$ (i.e., $\beta_{h}(\phi \cdot b)(x)=\phi(x \cdot h) \beta_{h}(b)(x)$ ),

$$
f(r h, x)(x)=\beta_{h}^{-1}(\Phi(f)(r))(x),
$$

and $\Phi$ is a $*$-homomorphism of $\operatorname{Ind}_{H}^{G \times X}(B, \beta)$ into $\operatorname{Ind}_{H}^{G}(B, \beta)$, which clearly has kernel $J$.

Finally, it is not difficult (cf., e.g., $\left[13\right.$, Lemma 2.6]) to see that $\Phi\left(\operatorname{Ind}_{H}^{G \times X}(B, \beta)\right)$ is a rich subalgebra of $\operatorname{Ind}_{H}^{G}(B, \beta)$ as defined in [4, Definition 11.1.1]. Thus $\Phi$ is surjective by [4, Lemma 11.1.4].

Corollary 10. Suppose that $X$ is a locally compact left $G$-space, and that $H$ is a closed normal subgroup of $G$ which acts freely and properly on $X$. Then there is an isomorphism of $\operatorname{Br}_{G / H}(H \backslash X)$ onto $\operatorname{Br}_{G}(X)$ taking $[B, \beta]$ to $\left[p^{*}(B), p^{*}(\beta)\right]=$ $\left[p^{*}(B), \tau \otimes \beta\right]$.

Proof. View $Y=X \times G / H$ as a left $G$-space via the diagonal action, and a right $G / H$-space via right translation on the second factor. Both actions are free, and the second action is proper. To see that the first action is proper, suppose that 
$\left(x_{n}, t_{n} H\right) \rightarrow(x, t H)$ while $\left(s_{n} \cdot x_{n}, s_{n} t_{n} H\right) \rightarrow(y, r H)$. Then $s_{n} H \rightarrow s H$ for some $s \in G$. Passing to a subsequence and relabeling, we can assume that there are $h_{n} \in H$ such that $h_{n} s_{n} \rightarrow s$ in $G$. But then $s_{n} \cdot x_{n} \rightarrow y$ while $h_{n} \cdot\left(s_{n} \cdot x_{n}\right) \rightarrow s \cdot x$. Since the $H$-action is proper, we can assume that $h_{n} \rightarrow h$ in $H$. Thus $s_{n} \rightarrow h^{-1} s$, and this proves the claim.

The map $G \cdot(x, t H) \mapsto H t^{-1} \cdot x$ is a bijection $\phi$ of $G \backslash Y$ onto $H \backslash X$. Further, $G \backslash Y$ is a right $G / H$-space and $H \backslash X$ is a left $G / H$-space with

$$
\phi\left(v \cdot\left(s^{-1} H\right)\right)=s H \cdot \phi(v) .
$$

(That is, $\phi$ is equivariant when the $G / H$-action on $G \backslash Y$ is viewed as a left-action.) Therefore,

$$
\operatorname{Br}_{G / H}(G \backslash Y) \cong \operatorname{Br}_{G / H}(H \backslash X) .
$$

Similarly, $Y /(G / H)$ and $X$ are isomorphic as left $G$-spaces so that

$$
\operatorname{Br}_{G}(Y /(G / H)) \cong \operatorname{Br}_{G}(X) .
$$

Finally, Theorem 1 implies that

$$
\operatorname{Br}_{G}(Y /(G / H)) \cong \operatorname{Br}_{G / H}(G \backslash Y)
$$

Thus, Equations (7)-(9) imply that there is an isomorphism of $\operatorname{Br}_{G / H}(H \backslash X)$ onto $\operatorname{Br}_{G}(X)$ sending $(B, \beta)$ to $\left(\operatorname{Ind}_{G / H}^{X \times G / H}(B, \beta) / J, \tau \otimes \mathrm{id}\right)$ with

$$
J=\left\{f \in \operatorname{Ind}_{G / H}^{X \times G / H}(B, \beta): f(x, r H)\left(H r^{-1} \cdot x\right)=0 \text { for all } x \in X\right\} .
$$

Define $\Phi: \operatorname{Ind}_{G / H}^{X \times G / H}(B, \beta) \rightarrow C_{0}(X, B)$ by $\Phi(f)(x)=f(x, H)$. Then $\Phi$ is onto (see, for example, the first sentence of the proof of [13, Lemma 2.6]). Since

$$
\begin{aligned}
\Phi\left(\tau_{s} \otimes \operatorname{id}(f)\right)(x) & =\tau_{s} \otimes \operatorname{id}(f)(x, H)=f\left(s^{-1} \cdot x, s^{-1} H\right) \\
& =\beta_{s H}\left(f\left(s^{-1} \cdot x, H\right)\right)=\tau_{s} \otimes \beta_{s H}(\Phi(f))(x),
\end{aligned}
$$

$\Phi$ is equivariant, and it only remains to show that $\Phi$ induces a bijection of the quotient by $J$ with the quotient of $C_{0}(X, B)$ by the balancing ideal $I$.

However, if $\Phi(f) \in I$, then $f(x, H)(H \cdot x)=0$ for all $x \in X$. But then $f(x, r H)\left(H r^{-1} \cdot x\right)=\beta_{r H}^{-1}(f(x, H))\left(H r^{-1} \cdot x\right)$, which is zero since $\beta$ covers the $G / H$-action on $X$, and $f \in J$. The argument reverses, so $\Phi(J)=I$, and the result follows.

\section{REFERENCES}

1. F. Combes, Crossed products and Morita equivalence, Proc. London Math. Soc. (3) 49 (1984), 289-306. MR 86c:46081

2. David Crocker, Alex Kumjian, Iain Raeburn, and Dana P. Williams, An equivariant Brauer group and actions of groups on $C^{*}$-algebras, preprint.

3. Raul E. Curto, Paul Muhly, and Dana P. Williams, Crossed products of strongly Morita equivalent $C^{*}$-algebras, Proc. Amer. Math. Soc. 90 (1984), 528-530. MR 85i:46083

4. Jacques Dixmier, $C^{*}$-algebras, North-Holland, New York, 1977. MR 56:16388

5. Edward Effros, Transformation groups and $C^{*}$-algebras, Ann. of Math. (2) 81 (1965), 38-55. MR 30:5175

6. J. M. G. Fell, An extension of Mackey's method to Banach *-algebraic bundles, Mem. Amer. Math. Soc. 90 (1969), 1-168. MR 41:4255

7. Philip Green, $C^{*}$-algebras of transformation groups with smooth orbit space, Pacific J. Math. 72 (1977), 71-97. MR 56:12170

8. The local structure of twisted covariance algebras, Acta Math. 140 (1978), 191-150. MR 58:12376 
9. Kjeld Knudsen Jensen and Klaus Thomsen, Elements of KK-theory, Birkhäuser, Boston, 1991. MR 94b:19008

10. Iain Raeburn, On the Picard group of a continuous-trace $C^{*}$-algebra, Trans. Amer. Math. Soc. 263 (1981), 183-205. MR 82b:46090

11. Induced $C^{*}$-algebras and a symmetric imprimitivity theorem, Math. Ann. 280 (1988), 369-387. MR 90k:46144

12. Iain Raeburn and Jonathan Rosenberg, Crossed products of continuous-trace $C^{*}$-algebras by smooth actions, Trans. Amer. Math. Soc. 305 (1988), 1-45. MR 89e:46077

13. Iain Raeburn and Dana P. Williams, Pull-backs of $C^{*}$-algebras and crossed products by certain diagonal actions, Trans. Amer. Math. Soc. 287 (1985), 755-777. MR 86m:46054

14. Marc A. Rieffel, Unitary representations of group extensions: An algebraic approach to the theory of Mackey and Blattner, Adv. in Math. Suppl. Stud. 4 (1979), 43-81. MR 81h:22004

15. Applications of strong Morita equivalence to transformation group $C^{*}$-algebras, Proc. Sympos. Pure Math., vol. 38, Part I, Amer. Math. Soc., Providence, RI, 1982, pp. 299-310. MR 84k:46046

Department of Mathematics, University of Nevada, Reno, Nevada 89557

E-mail address: alex@math.unr.edu

Department of Mathematics, University of Newcastle, Newcastle, New South Wales 2308, Australia

E-mail address: iain@math.newcastle.edu.au

Department of Mathematics, Dartmouth College, Hanover, New Hampshire 037553551

E-mail address: dana.williams@dartmouth.edu 